\title{
GESTÃO DE RECURSOS HUMANOS EM UMA EMPRESA DE TELECOMUNICAÇÕES - UM ENFOQUE DA ESCOLA FRANCESA DO APOIO MULTICRITÉRIO À DECISÃO
}

\author{
Laurent Lacaze ${ }^{1}$ \\ Luiz Flavio Autran Monteiro Gomes ${ }^{2}$ \\ Maria Augusta Soares Machado ${ }^{3}$
}

\begin{abstract}
Resumo: Este artigo apresenta um estudo de caso desenvolvido numa importante operadora de telefonia brasileira no âmbito do planejamento anual de seus custos com pessoal. Através da aplicação da metodologia de Apoio Multicritério à Decisão ELECTRE TRI, o estudo objetivou racionalizar a alocação de recursos atribuindo soluções, ou recomendações, diferenciadas aos diversos setores da companhia de modo a satisfazer uma ampla gama de objetivos. O principal interesse do trabalho consiste em demonstrar como aprimorar um processo decisório através de uma metodologia de agregação multicritério sem critério único de síntese por meio de um procedimento de triagem. Os resultados alcançados evidenciam os ganhos obtidos com o desenvolvimento do estudo e destacam os benefícios proporcionados pela utilização da técnica empregada. Com a participação de diversos profissionais provenientes de vários órgãos da empresa, as decisões tomadas durante o planejamento tornaram-se mais transparentes e consistentes, contribuindo para a geração de um clima de entendimento e compromisso, melhorando a credibilidade do processo. O artigo é iniciado com uma seção introdutória. Posteriormente, é apresentada uma descrição da metodologia empregada e suas principais características. Na seqüência, a descrição do estudo de caso é abordada a partir de suas principais etapas. Por fim, o estudo é concluído com a descrição dos benefícios proporcionados pelo trabalho, assim como de recomendações para pesquisas futuras.
\end{abstract}

Palavras-Chave: Estudo de Caso, Apoio Multicritério à Decisão, ELECTRE TRI.

\begin{abstract}
This work presents a study carried out in an important firm of telecommunications as part of its corporate planning. Based on a diagnosis made within the area of human resources, important opportunities were found to improve the process whereby budgetted labor expenses of the company were planned. Starting from the fundamental objective of making the best of the allocation of the resources, a thorough investigation was carried out of the methods to be used to ensure that decisions reached had been fully studied and relevant factors taken into consideration. In accordance with the characteristics of the decision process and the variety of the objectives to be taken into consideration, it was decided to use the methods of Multi-Criteria Decision Making known as ELECTRE TRI in order to comprehend the different dimensions which make up the problem and to contribute to the process of communication between the department of the company. In accordance with the strategy of offering solutions whose costs are in line with the needs and characteristics of the areas, the present study covers a system of methods which presents solutions based on the understanding of the many figures which make up the basic profile of each unit of the firm. In this way, the human resources area, can offer varying recommendations and in a significant way improve the logic and clarity of the process. After undertaking severe examination, the study shows a balance sheet that confirms the significant benefits obtained from the study.
\end{abstract}

Key words: Case Study, Multi-Criteria Decision Making, ELECTRE TRI.

$\overline{\text { 1,2,3 } \text { Faculdades Ibmec-RJ, E-mail: }{ }^{1}}$ laurent@samerica.com, ${ }^{2}$ autran@ibmecrj.br, ${ }^{3}$ mmachado@ibmecrj.br 


\section{INTRODUÇÃO}

O presente estudo foi desenvolvido e aplicado em uma empresa brasileira de telecomunicações, sediada no Rio de Janeiro e composta por mais de $12 \mathrm{mil}$ funcionários. O seu principal objetivo foi apoiar a área de recursos humanos (RH) da companhia no planejamento dos seus custos com mão-de-obra realizado na ocasião do planejamento corporativo anual para o exercício de 2003. Buscouse, fundamentalmente, otimizar a alocação destes recursos a partir da utilização de metodologia de Apoio Multicritério à Decisão (AMD).

Os custos com pessoal são definidos e gerenciados pela Diretoria de $\mathrm{RH}$ a partir das orientações e premissas advindas do planejamento estratégico da companhia. Eles compõem os denominados custos e despesas operacionais da empresa e são devidamente reportados (no caso das companhias de capital aberto - S.A.) nos demonstrativos de resultados e relatórios anuais das empresas. Eles são compostos pelos salários dos empregados, benefícios, adicionais e encargos sociais e trabalhistas, dentre outros.

A utilização de técnicas de AMD proporcionou consistência e lógica ao processo e foi motivada pela necessidade de estruturar a ampla gama de informações de $\mathrm{RH}$ de maneira a se delinear melhor as características e peculiaridades dos órgãos da companhia. Ou seja, era preciso determinar como os recursos seriam alocados considerando-se as carências e necessidades de cada unidade.

Por outro lado, existia a necessidade de traduzir-se por meio de uma estrutura hierárquica, os principais desafios da companhia no contexto da decisão. Com efeito, a área de $\mathrm{RH}$ precisava de um instrumento analítico que facilitasse a organização de valores e objetivos, de modo a determinar como e onde tais recursos estariam sendo alocados.

Ademais, o trabalho demonstrou como as técnicas de AMD podem ser eficazmente utilizadas para o gerenciamento do capital humano das organizações.

\section{A ESCOLHA DO MODELO DE APOIO MULTICRITÉRIO À DECISÃO ADOTADO}

Segundo Larichev e Olson (2001), “os estudos comparativos entre as diversas modalidades de $A M D$, demonstram que não existe nenhuma metodologia que se destaca das outras em todos os contextos de decisão envolvendo múltiplos critérios. Um analista de decisão deve possuir conhecimentos suficientes para determinar qual a melhor metodologia a ser aplicada em função das características da decisão que deve ser tomada".

Para determinar a metodologia de AMD mais adequada para o contexto de decisão, empreendeu-se uma ampla investigação dentre as diversas metodologias de AMD. Esta investigação se concentrou essencialmente nas características do problema. A partir da análise das fontes de dados que iriam subsidiar os atributos do modelo decisório, constatou-se a existência de diversos critérios de ordem qualitativa. Em função disso, inclinou-se pela utilização dos métodos ELECTRE (Élimination et Choix Traduisant la Réalité. Roy, 1968) visto que são mais adequados para os problemas onde a natureza dos critérios é fortemente heterogênea, onde as performances (ou avaliações) das alternativas podem ser efetuadas nas mais diferentes escalas e unidades. Adicionalmente a este fato, prevaleceu a opinião de que a agregação multicritério sem critério único de síntese apresenta não uma deficiência, mas sim uma vantagem na análise de decisão. A imposição de uma pré-ordem completa conduz freqüentemente a uma modelagem de preferências pouco realista. Ao permitir quatro diferentes formas de comparação entre alternativas, os métodos baseados em técnicas de superação oferecem sem dúvida uma importante contribuição para o agente de decisão.

Portanto, o estudo adotou a metodologia 
ELECTRE TRI ${ }^{2}$, pertencente à Escola Francesa de AMD, que se baseia na noção de superação (surclassement). Esta metodologia aplica-se à problemática P. $\beta$, que tem por objetivo esclarecer a decisão por uma triagem resultante da alocação de cada alternativa (ou ação) a uma categoria (ou classe). As diferentes categorias são definidas a priori com base em normas aplicáveis ao conjunto de alternativas por meio da comparação de cada alternativa potencial com uma referência estável (padrão/ alternativa de referência). Por conseguinte, a filosofia subjacente ao ELECTRE TRI consiste em classificar-se as alternativas, a partir de categorias definidas a priori, e sempre respeitando o conceito básico da Escola Francesa de AMD, que é o de superação, a seguir enunciado:

Diz-se que uma alternativa a supera outra alternativa b (simbolizando-se por a $\mathrm{S}$ b) se e somente se existem razões claras e positivas que justifiquem seja uma preferência, seja uma presunção de preferência em favor de uma (bem identificada) das duas ações, mas sem que nenhuma separação significativa seja estabelecida entre as situações de preferência estrita (P), de preferência fraca $(\mathrm{Q})$ e de indiferença (I).

$$
\text { a S b } \Leftrightarrow(a \mathrm{P} b \text { ou a Q b ou a I b) }
$$

(Roy e Bouyssou, 1993). Uma apresentação dos métodos ELECTRE e de outros da Escola Francesa do Apoio Multicritério à Decisão também pode ser encontrada em Gomes et al. (2003), pgs. 93-136.

A alocação de uma alternativa $a_{k}$ em uma categoria é fruto do resultado da avaliação de $a_{k}$ a partir dos critérios e das normas que definem tal categoria. Tal alocação não influencia as categorias em que as outras alternativas serão alocadas. O método ELECTRE TRI aloca as ações nas categorias a partir de duas etapas distintas: (1) construção de uma relação

\footnotetext{
${ }^{2}$ Para maiores informações sobre a metodologia, consultar Yu (1992).

ENGEVISTA, v. 6, n. 1, p. 4-18, abr. 2004
}

de superação $S$ que estabelece como as alternativas se enquadram nos limites de cada categoria; (2) exploração da relação de superação $S$ de modo a alocar as alternativas nas suas respectivas categorias. Cinco categorias foram consideradas no estudo, diferenciadas com base nas estratégias de investimento destinadas às unidades que forem compôlas.

\section{ESTUDO DE CASO}

\subsection{Análise das Conseqüências e Elaboração dos Critérios}

Independentemente da maneira pela qual elaboramos a solução de um problema, ou sobre a recomendação de um rumo de ação diante de uma situação bem definida, é importante empreender uma análise, avaliação e ponderação das conseqüências associadas às alternativas factíveis de serem escolhidas. Tais conseqüências são, de modo geral, múltiplas e compreendem unidades, ou medidas de valor, das mais diversas. $\mathrm{Na}$ medida em que as conseqüências das ações são consideradas e avaliadas, os agentes envolvidos na decisão podem comparar as alternativas com base num modelo de preferências.

A partir deste entendimento, os profissionais envolvidos no trabalho passaram a elaborar um modelo esquemático com o objetivo de avaliar e comparar as características e necessidades de cada unidade da empresa a partir de suas performances nos critérios que iriam subsidiar o estudo. A construção deste modelo serviu para, numa próxima etapa, modelar as preferências globais constituídas pela importância relativa destes critérios assim como pelas suas dimensões. É importante destacar que o método ELECTRE TRI é aplicado com base em preferências formalizadas a partir da construção de uma família coerente de critérios.

Diante desta perspectiva, algumas questões foram colocadas a fim de nortear a identificação das conseqüências: (1) quais conseqüências 
são suscetíveis de interferir nos objetivos ou sistemas de valor considerados na processo decisório?; (2) dentre as conseqüências consideradas, quais devem ser formalizadas e como? (3) até que ponto fatores como imprecisão e incerteza, podem, eventualmente, interferir na avaliação de tais conseqüências? A consideração destas questões serviu para nortear esta etapa do trabalho e, assim, fundamentar a formação da família de critérios adotada neste estudo.

Foi, portanto, com base nestas considerações, que se levantou uma primeira lista de conseqüências elementares - representadas pelas características ou atributos das unidades da organização - que deveriam constituir o espectro de conseqüências do estudo. Uma definição foi associada a cada conseqüência a fim de possibilitar que todos os envolvidos no processo decisório pudessem compreendê-las de forma clara e inequívoca. Após testes de coerência, esta lista, julgada exaustiva, foi finalmente constituída por 13 conseqüências elementares que determinaram a maneira pela qual cada unidade da organização passou a ser avaliada, conforme apresentado na tabela 1.

Tabela 1: Conseqüências Elementares

\begin{tabular}{|c|l|}
\hline & Dimensão \\
\hline 1 & Posicionamento estratégico das unidades \\
\hline 2 & Avaliação de desempenho das unidades \\
\hline 3 & Avaliação de Desempenho dos empregados \\
\hline 4 & Estrutura hierárquica das áreas \\
\hline 5 & Categorias funcionais dos empregados \\
\hline 6 & Taxa de reajuste salarial frente à composição salarial das unidades \\
\hline 7 & Reajustes salariais pelo headcount médio das unidades \\
\hline 8 & Formação acadêmica \\
\hline 9 & Qualificação dos cargos \\
\hline 10 & Análise discriminante dos empregados frente ao turnover voluntário \\
\hline 11 & Índices de turnover voluntário \\
\hline 12 & Eqüidade salarial externa \\
\hline 13 & Eqüidade salarial interna \\
\hline
\end{tabular}

Portanto, esta lista de conseqüências elementares representou a forma pela qual os órgãos da companhia foram analisados e avaliados, no sentido de fundamentar um julgamento claro e estruturado de suas necessidades e características principais. Para que as conseqüências pudessem ser vistas como dimensões, associou-se uma escala de preferência (ou vetor de performance) a cada uma delas, de forma a determinar o julgamento preciso da sua performance. Com isso, as conseqüências levantadas passaram a representar as dimensões do problema ( $\mathrm{i}=13$ ), abrindo caminho para ENGEVISTA, v. 6, n. 1, p. 4-18, abr. 2004 se iniciar o processo de constituição dos critérios do modelo decisório.

Segundo Roy e Bouyssou (1993), "um critério visa resumir, a partir de uma função, as avaliações de uma ação (ou alternativa) sobre diversas dimensões podendo estar associadas a um mesmo eixo de significância”. Um critério pode ser entendido como sendo um "ponto de vista” ou "eixo de significância”, constituído a partir de uma ou várias dimensões. É a partir do conjunto de critérios do modelo decisório que os agentes envolvidos na construção do modelo decisório podem formalizar as 
suas preferências de maneira global. Este conjunto passa a representar uma família, designada por $\mathrm{F}$, que deve possuir uma coerência mínima definida a partir de axiomas operacionais.

Portanto, as treze dimensões identificadas serviram de base para a formação de uma família de critérios. É importante observar que, a rigor, cada dimensão pode constituir um critério. Não obstante, a análise privilegiou a formação de critérios que representassem preocupações elementares no contexto do trabalho.

Alguns princípios foram seguidos na definição dos critérios: (1) o eixo de significância sobre o qual cada critério agrega as performances das dimensões, deve ser facilmente compreendido por todas as pessoas envolvidas no processo decisório; (2) o número de critérios deve ser o menor possível a fim de evitar contestações e facilitar o entendimento dos mesmos; (3) a família de critérios deve ser aceita como base para a aferição das performances das unidades da companhia de modo a possibilitar comparações e justificar as preferências globais assumidas no estudo.

Diversos agrupamentos de dimensões foram testados segundo estes princípios. Finalmente, após a aplicação de testes de coerência, uma família de 7 critérios foi estabelecida. Com isso, chegou-se ao quadro da tabela 2, que formalizou 0 espectro de conseqüências (também denominado nuvem de conseqüências v(a))- representado pela família $F$ de critérios: $g_{1}, g_{2}, \ldots, g_{7}$.

Tabela 2: Critérios

\begin{tabular}{|l|}
\hline Critérios \\
\hline $\mathrm{g}_{1}$ - Posicionamento Estratégico \\
\hline $\mathrm{g}_{2}$ - Avaliação de Desempenho \\
\hline $\mathrm{g}_{3}$ - Estrutura da Unidade \\
\hline $\mathrm{g}_{4}$ - Histórico de Progressões Salariais \\
\hline $\mathrm{g}_{5}-$ Qualificação Profissional \\
\hline $\mathrm{g}_{6}-$ Turnover Voluntário \\
\hline $\mathrm{g}_{7}-$ Eqüidades Salariais \\
\hline
\end{tabular}

\subsection{Axiomas Fundamentais de Coerência e Testes Operacionais}

Para que uma família de critérios $\mathrm{F}$ possa desempenhar adequadamente sua função de apoiar um processo decisório estabelecendo preferências sobre um conjunto de ações potenciais, três axiomas básicos de coerência precisam ser respeitados. São eles: axiomas da exaustividade, da coesão e da não redundância.

Após a identificação das dimensões, os analistas passaram a aplicar os testes de coerência a fim de assegurar que os critérios de $\mathrm{F}$ se associem a um modelo de preferências restrito. $\mathrm{O}$ seu conjunto ENGEVISTA, v. 6, n. 1, p. 4-18, abr. 2004 deve permitir a modelagem de preferências do problema em um

contexto global. Procurou-se, portanto, garantir que a família $\mathrm{F}$ assegure uma coerência mínima entre estes dois níveis. Os resultados advindos dos testes operacionais de coerência estão sintéticamente expostos nesta seção.

- Axioma fundamental da exaustividade: $\mathrm{O}$ critério $\mathrm{g}_{7}$ (Eqüidades Salariais), originalmente considerava apenas as comparações com as outras empresas participantes das pesquisas salariais efetuadas pela 
companhia. Notou-se que além desta consistência externa, era necessário efetuar uma comparação interna, a partir das unidades da própria empresa. Desta forma, adotou-se mais uma dimensão: "Eqüidade Salarial Interna" para refletir de maneira fidedigna 0 modelo de preferências ligado ao seu eixo de significância.

- Axioma fundamental da coesão: Todos os critérios considerados no modelo de preferências passaram no teste.

- Axioma fundamental da nãoredundância: $\mathrm{Na}$ formação do critério $g_{3}$ (Estrutura das Unidade), considerou-se inicialmente uma dimensão que, posteriormente, não foi validada no teste da não redundância. O teste permitiu verificar que as outras dimensões do critério, anulavam a necessidade de sua existência, visto que garantiam a apreensão integral das conseqüências do modelo de preferências estruturado.

Portanto, após os testes de coerência, a família coerente de critérios (F.C.C.) adotada no estudo foi representada por $F=\left\{g_{1}, g_{2}, g_{3}, g_{4}, g_{5}, g_{6}, g_{7}\right\}$, definida em A. Tal família acha-se apresentada na tabela 3.

Tabela 3: Família Coerente de Critérios

\begin{tabular}{|c|c|}
\hline Critérios & Dimensões \\
\hline$g_{1}$ - Posicionamento Estratégico & $\begin{array}{l}\text { Posicionamento estratégico das } \\
\text { unidades }\end{array}$ \\
\hline $\mathrm{g}_{2}$ - Avaliação de Desempenho & $\begin{array}{l}\text { Avaliação de desempenho das unidades; } \\
\text { Avaliação de desempenho dos } \\
\text { empregados }\end{array}$ \\
\hline $\mathrm{g}_{3}$ - Estrutura da Unidade & $\begin{array}{l}\text { Estrutura hierárquica das Áreas; } \\
\text { Categorias funcionais dos Empregados }\end{array}$ \\
\hline $\begin{array}{l}\mathrm{g}_{4} \text { - Histórico de Progressões } \\
\text { Salariais }\end{array}$ & $\begin{array}{l}\text { Taxa de reajuste frente à composição } \\
\text { salarial das unidades; } N^{0} \text { de reajustes } \\
\text { pelo headcount médio das unidades }\end{array}$ \\
\hline$g_{5}$ - Qualificação Profissional & $\begin{array}{l}\text { Formação Acadêmica; Qualificação dos } \\
\text { cargos }\end{array}$ \\
\hline$g_{6}$ - Turnover Voluntário & $\begin{array}{l}\text { Análise discriminante dos perfis dos } \\
\text { empregados frente ao turnover } \\
\text { voluntário; Índices de turnover } \\
\text { voluntário }\end{array}$ \\
\hline g7 - Eqüidades Salariais & Eqüidade salarial interna; externa \\
\hline
\end{tabular}

\subsection{Matriz de Performance e Definição dos Pseudo-critérios}

Após a definição da família de critérios, passou-se à coleta de dados de forma a permitir a construção da Matriz de Performance relacionando o desempenho de cada unidade da empresa aos critérios do estudo. Esta avaliação é feita com base nas escalas de preferência relativas ENGEVISTA, v. 6, n. 1, p. 4-18, abr. 2004 às 13 dimensões nas quais os critérios foram embasados. É importante sinalizar que tais avaliações foram, em certas dimensões, pontuais (dimensões 1, 6, 7, 11 e 13). Para as demais, adotou-se a técnica de pontualização por média ponderada. Assim sendo, pôde-se chegar finalmente à matriz que relaciona $o$ desempenho das 13 unidades da 
Tabela 4: Matriz de Performance

\begin{tabular}{|c|c|c|c|l|l|l|l|}
\hline $\begin{array}{c}\text { Critérios } \\
/ \\
\text { Unidaded }\end{array}$ & $\begin{array}{l}\mathrm{g}_{1}: \\
\text { Posiciona- } \\
\text { mento } \\
\text { Estratégico }\end{array}$ & $\begin{array}{l}\mathrm{g}_{2}: \\
\text { Avaliação } \\
\text { de Desem- } \\
\text { penho }\end{array}$ & $\begin{array}{l}\mathbf{g}_{3}: \\
\text { Estrutura } \\
\text { da } \\
\text { Unidade }\end{array}$ & $\begin{array}{l}\mathrm{g}_{4}: \\
\text { Histórico de } \\
\text { Progressão } \\
\text { Salarial }\end{array}$ & $\begin{array}{l}\mathrm{g}_{5}: \\
\text { Qualificação } \\
\text { Profissional }\end{array}$ & $\begin{array}{l}\mathrm{g}_{6}: \\
\text { Turnover } \\
\text { Voluntário }\end{array}$ & $\begin{array}{l}\mathrm{g}_{7}: \\
\text { Eqüidades } \\
\text { Salariais }\end{array}$ \\
\hline $\mathbf{a}_{\mathbf{1}}$ & 1.0 & 4.0 & 4.6 & 6.0 & 4.3 & 2.7 & 5.5 \\
\hline $\mathbf{a}_{\mathbf{2}}$ & 1.0 & 5.0 & 4.9 & 2.0 & 5.0 & 1.3 & 8.2 \\
\hline $\mathbf{a}_{\mathbf{3}}$ & 3.0 & 4.5 & 5.7 & 3.0 & 4.4 & 9.7 & 6.0 \\
\hline $\mathbf{a}_{\mathbf{4}}$ & 1.0 & 3.5 & 3.4 & 7.0 & 4.1 & 4.7 & 6.5 \\
\hline $\mathbf{a}_{\mathbf{5}}$ & 1.0 & 2.0 & 5.1 & 7.0 & 4.9 & 3.7 & 8.2 \\
\hline $\mathbf{a}_{\mathbf{6}}$ & 3.0 & 6.5 & 4.3 & 5.0 & 5.2 & 12.0 & 8.0 \\
\hline $\mathbf{a}_{\mathbf{7}}$ & 3.0 & 5.5 & 5.7 & 5.0 & 5.7 & 12.2 & 8.9 \\
\hline $\mathbf{a}_{\mathbf{8}}$ & 3.0 & 7.0 & 5.4 & 1.0 & 5.4 & 1.9 & 5.9 \\
\hline $\mathbf{a}_{\mathbf{9}}$ & 3.0 & 4.0 & 6.2 & 6.0 & 5.2 & 11.6 & 4.9 \\
\hline $\mathbf{a}_{\mathbf{1 0}}$ & 2.0 & 3.0 & 4.3 & 9.0 & 3.5 & 2.8 & 8.2 \\
\hline $\mathbf{a}_{\mathbf{1 1}}$ & 3.0 & 5.5 & 6.4 & 9.0 & 6.0 & 3.5 & 4.6 \\
\hline $\mathbf{a}_{\mathbf{1 2}}$ & 2.0 & 5.0 & 5.0 & 3.0 & 5.1 & 3.6 & 6.2 \\
\hline $\mathbf{a}_{\mathbf{1 3}}$ & 3.0 & 7.0 & 6.3 & 3.0 & 5.7 & 2.1 & 4.8 \\
\hline
\end{tabular}

De forma a determina-se valores dos pseudo-critérios compostos pelos limiares de preferência fraca $\left(q_{j}\right)$ e forte $\left(p_{j}\right)$, finalizou-se primeiro a matriz de performance. Deste modo, pôde-se identificar e analisar de maneira mais realista os desvios encontrados no desempenho das unidades sob os diferentes critérios. Ou seja, considerando-se os valores encontrados e levando-se em conta fatores como imprecisão, incerteza e indeterminação, iniciou-se uma discussão sobre as diferenças $g_{j}\left(a_{k}\right)-g_{j}\left(a_{i}\right)$, que poderiam fundamentar as limiares de preferência fraca e forte. Foi com base neste procedimento que se chegou finalmente aos valores dos limiares para $q_{j}$ e $p_{j}$, conforme apresentado na tabela 5.

Tabela 5: Valores para $\mathbf{q}_{\mathbf{j}} \mathbf{e} \mathbf{p}_{\mathbf{j}}$

\begin{tabular}{|c|c|c|c|c|c|c|c|}
\cline { 2 - 7 } \multicolumn{1}{c|}{} & $\mathbf{g}_{\mathbf{1}}$ & $\mathbf{g}_{\mathbf{2}}$ & $\mathbf{g}_{\mathbf{3}}$ & $\mathbf{g}_{\mathbf{4}}$ & $\mathbf{g}_{\mathbf{5}}$ & $\mathbf{g}_{\mathbf{6}}$ & $\mathbf{g}_{\mathbf{7}}$ \\
\hline $\mathbf{q}_{\mathbf{j}}$ & 1 & 1 & 0.5 & 1 & 0.6 & 1 & 1 \\
\hline $\mathbf{p}_{\mathbf{j}}$ & 1 & 2.5 & 1.3 & 2 & 1.3 & 2.5 & 1.5 \\
\hline
\end{tabular}

\subsection{Modelagem e Exploração do ELECTRE TRI}

A aplicação da metodologia ELECTRE TRI foi realizada em quatro etapas distintas: 
1. atribuição de valores aos pesos $\mathrm{k}_{\mathrm{j}}$;

2. atribuição de valores aos limiares de veto $\mathrm{v}_{\mathrm{j}}$;

3. exploração da relação $S$ do ELECTRE TRI;

4. análises de sensibilidade e de robustez

\subsubsection{Atribuição de Valores a $\mathbf{k}_{\mathbf{j}}$}

$\mathrm{O}$ procedimento adotado no que tange a determinação do peso dos critérios $\left(\mathrm{k}_{\mathrm{j}}\right)$, não consistiu na atribuição direta dos pesos dos critérios. Evitou-se a definição de $\mathrm{k}_{\mathrm{j}}$ de forma absoluta, desconsiderando o fato de que a família de critérios consiste em um sistema de preferências que, a despeito do papel que cada critério exerce, não pressupõe que os seus julgamentos de valor sejam impostos de forma isolada. Desta maneira, a importância acordada a cada critério não foi efetuada sem a comparação direta com aqueles outros que compõem $F$. A partir de uma abordagem construtivista, formalizou-se diversas hipóteses na tentativa de representar de forma mais fidedigna possível, o sistema de valores das pessoas envolvidas no trabalho. Com isso, a construção dos valores de $\mathrm{k}_{\mathrm{j}}$ deuse a partir de ajustamentos, até que fosse determinada a importância relativa dos critérios.

A técnica utilizada foi a de sistemas de inequações. Esta forma de proceder procurou refletir as opiniões de ordem qualitativa sobre a importância que convém atribuir a cada critério. Deste modo, criou-se consenso a partir das seguintes inequações:

$$
\begin{array}{ll}
\text { - } \mathrm{k}_{1}>\mathrm{k}_{2}>\mathrm{k}_{4}=\mathrm{k}_{7}>\mathrm{k}_{6}= \\
\text { - } \mathrm{k}_{3}>\mathrm{k}_{5} \\
\text { - } \mathrm{k}_{1}=\mathrm{k}_{2}+\mathrm{k}_{4} \\
\text { - } \mathrm{k}_{2}=\mathrm{k}_{4}+1 \\
\text { - } \mathrm{k}_{4}=\mathrm{k}_{6}+2 \mathrm{k}_{5} \\
\mathrm{k}_{6}=3 \mathrm{k}_{5}
\end{array}
$$

Presumindo-se de forma não restritiva que $k_{5}=1$, chega-se, finalmente, aos valores apresentados na tabela 6 .

\section{Tabela 6: Valores para $\mathbf{k}_{\mathbf{j}}$}

\begin{tabular}{|c|c|c|c|c|c|c|c|}
\cline { 2 - 8 } \multicolumn{1}{c|}{} & $\mathbf{g}_{\mathbf{1}}$ & $\mathbf{g}_{\mathbf{2}}$ & $\mathbf{g}_{\mathbf{3}}$ & $\mathbf{g}_{\mathbf{4}}$ & $\mathbf{g}_{\mathbf{5}}$ & $\mathbf{g}_{\mathbf{6}}$ & $\mathbf{g}_{\mathbf{7}}$ \\
\hline $\mathbf{k}_{\mathbf{j}}$ & 11 & 6 & 3 & 5 & 1 & 3 & 5 \\
\hline
\end{tabular}

\subsubsection{Atribuição de Valores aos Limiares de Veto $\mathbf{v}_{\mathbf{j}}$}

As análises empreendidas no estudo para se determinar os limiares de veto $\left(\mathrm{v}_{\mathrm{j}}\right)$ levaram em conta os valores atribuídos a $\mathrm{k}_{\mathrm{j}}$. Ou seja, foi decidido que os valores de veto deveriam ser definidos tendo em vista a importância relativa dos critérios. A principal questão levantada consistiu em julgar se era conveniente, para $\mathrm{g}_{\mathrm{j}}$, a possibilidade de impor o poder de veto, considerando-se a hipótese de superação em todos os outros critérios. Desta forma, julgou-se pertinente conceber $\mathrm{v}_{\mathrm{j}}$ como dependente de $g_{j}$.

Um parâmetro importante para a atribuição dos valores de $\mathrm{V}_{\mathrm{j}}$ consistiu, para cada critério considerado, na relação definida por $\mathrm{v}_{\mathrm{j}} / \mathrm{p}_{\mathrm{j}}\left(\right.$ com $\left.\mathrm{p}_{\mathrm{j}} \neq 0\right)$. Com isso, pôde-se determinar que os critérios representados por $g_{5}$ e $g_{3}$, não deveriam impor a possibilidade de veto em função das características peculiares de tais critérios. Deste modo, atribuiu-se valores extremos para $\mathrm{v}_{5}$ e $\mathrm{v}_{3}$, de tal sorte a não possibilitar a hipótese de veto. Os valores determinados para $\mathrm{v}_{\mathrm{j}}$ estão apresentados na tabela 7.

Tabela 7: Valores para $\mathbf{v}_{\mathbf{j}}$

\begin{tabular}{|c|c|c|c|c|c|c|c|}
\cline { 2 - 8 } \multicolumn{1}{c|}{} & $\mathbf{g}_{\mathbf{1}}$ & $\mathbf{g}_{\mathbf{2}}$ & $\mathbf{g}_{\mathbf{3}}$ & $\mathbf{g}_{\mathbf{4}}$ & $\mathbf{g}_{\mathbf{5}}$ & $\mathbf{g}_{\mathbf{6}}$ & $\mathbf{g}_{\mathbf{7}}$ \\
\hline $\mathbf{v}_{\mathbf{j}}$ & 2 & 5 & 4 & 6 & 3 & 7 & 4 \\
\hline
\end{tabular}




\subsection{Exploração da Relação $S$ do ELECTRE TRI}

A metodologia ELECTRE TRI destina-se à problemática P. $\beta$, que tem por objetivo esclarecer a decisão por uma triagem resultante da alocação de cada ação $a$ (representado por uma unidade da companhia) à uma categoria. Este procedimento de alocação (ou classificação) é realizado a partir de comparações entre as ações de A, e ações de referência, às quais denominamos $b_{h}$. Estas ações de referência são definidas para fundamentar a alocação das ações de A nas categorias estabelecidas no modelo. Cada ação de referência exerceu no contexto do estudo o papel de um órgão fictício, formalizando as características que uma unidade deveria possuir para fundamentar a sua alocação em uma categoria. As performances destes perfis marcam as fronteiras entre as categorias. Ou seja, um determinado perfil determina a fronteira entre duas categorias.

Adotou-se para este trabalho, cinco categorias distintas nas quais as unidades deveriam ser alocadas. Desta forma, apenas quatro ações de referência foram necessárias para se estabelecer os limites destas categorias. A ação $b_{1}$, por exemplo, determinou o limite entre as categorias $E$ e $D$, enquanto que a $b_{2}$, as categorias $D$ e $C$, e assim sucessivamente. Portanto, cada ação de referência $b_{h}$ limita na parte inferior, a categoria $\mathrm{C}_{\mathrm{h}+1}$ (denominado de perfil baixo), e na parte superior a categoria $\mathrm{C}_{h}$ (denominado de perfil alto).

Para chegar ao procedimento de alocação, os profissionais que participaram do trabalho passaram a investigar o perfil que pudesse ser considerado padrão para cada ação de referência. A definição deste perfil foi estabelecida a partir de seu desempenho nos $n$ critérios de F:

$\mathrm{g}\left(\mathrm{b}_{\mathrm{h}}\right)=\left(\mathrm{g}_{1}\left(\mathrm{~b}_{\mathrm{h}}\right), \ldots, \mathrm{g}_{\mathrm{n}}\left(\mathrm{b}_{\mathrm{h}}\right)\right)$

Após a construção de diversas hipóteses, chegou-se finalmente a um modelo que se julgou adequado para a determinação dos perfis de $b_{h}$, conforme apresentado na tabela 8. Cabe mencionar que uma importante preocupação no desenvolvimento dos perfis de referências das categorias foi concedida aos espaçamentos entre a performance das ações. Ou seja, na hipótese de duas ações de referência possuírem desempenhos relativamente semelhantes, pode ocorrer a possibilidade de dupla alocação. Para eliminar tal possibilidade, deve-se definir os perfis de $b_{h}$ com performances consistentemente diferentes no sentido de anular a possibilidade de ocorrer a alocação de uma determinada ação em duas categorias distintas.

\section{Tabela 8: Quadro dos Perfis de Referência $b_{h}$}

\begin{tabular}{|c|c|c|c|c|c|c|c|}
\cline { 2 - 8 } \multicolumn{1}{c|}{} & $\mathbf{g}_{\mathbf{1}}$ & $\mathbf{g}_{\mathbf{2}}$ & $\mathbf{g}_{\mathbf{3}}$ & $\mathbf{g}_{\mathbf{4}}$ & $\mathbf{g}_{\mathbf{5}}$ & $\mathbf{g}_{\mathbf{6}}$ & $\mathbf{g}_{\mathbf{7}}$ \\
\hline $\mathbf{b}_{\mathbf{1}}$ & 1 & 3 & 4 & 3 & 4 & 2 & 5 \\
\hline $\mathbf{b}_{\mathbf{2}}$ & 1 & 4 & 5 & 4 & 5 & 3 & 6 \\
\hline $\mathbf{b}_{\mathbf{3}}$ & 2 & 5 & 6 & 6 & 5 & 5 & 8 \\
\hline $\mathbf{b}_{\mathbf{4}}$ & 3 & 7 & 6 & 8 & 6 & 7 & 9 \\
\hline
\end{tabular}

Após a definição dos perfis de referência, passou-se a etapa da definição das relações binárias definidas genericamente por a $\mathrm{H}$ b. $\mathrm{O}$ passos e as fórmulas utilizadas nesta etapa foram os abaixo relacionados.

(1) cálculo do índice de concordância parcial:

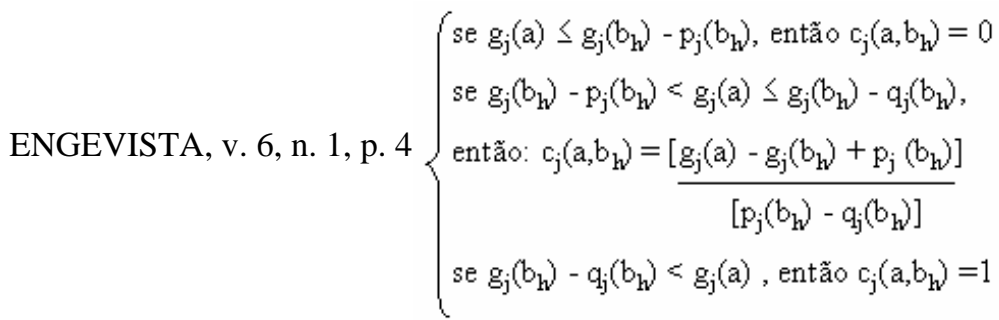


(2) cálculo do índice de concordância global:

$$
c\left(a, b_{h}\right)=\frac{\sum_{j \in F} k_{j} \cdot c_{j}\left(a, b_{h}\right)}{\sum_{j \in F} k_{j}}
$$

(3) cálculo do índice de discordância:

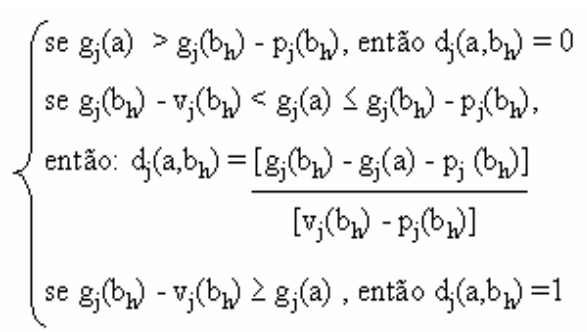

(4) cálculo do índice de credibilidade:

$$
\sigma\left(a, b_{b}\right)=c\left(a, b_{b}\right) \Pi \frac{1-d_{j}\left(a, b_{h}\right)}{1-c\left(a, b_{h}\right)} \operatorname{com} F=\left\{j \in F / d_{j}\left(a, b_{b}\right)>c\left(a, b_{h}\right)\right\}
$$

(5) definição das relações de preferência a partir da determinação do nível de corte $(\lambda)$.

Após testes operacionais, determinou-se que 0 nível de corte $\lambda$ inicialmente adotado deveria ser de 0,8. Com base nele, pôde-se finalmente determinar as relações binárias apresentadas na tabela 9, identificando as relações de superação

\begin{tabular}{|c|c|c|c|}
\hline \multicolumn{2}{|c|}{$\mathrm{a}_{\mathbf{k}} \mathrm{Sb}_{1}$} & \multicolumn{2}{|r|}{$\mathbf{a}_{\mathbf{k}} \mathbf{S b}_{2}$} \\
\hline $\mathrm{a}_{1} \mathrm{Ib}_{1}$ & $\mathrm{a}_{8} \mathrm{Sb}_{1}$ & $\mathrm{a}_{1} \mathrm{Ib}_{2}$ & $\mathrm{a}_{8} \mathrm{Sb}_{2}$ \\
\hline $\mathrm{a}_{2} \mathrm{Sb}_{1}$ & $\mathrm{a}_{9} \mathrm{Sb}_{1}$ & $\mathrm{a}_{2} \mathrm{Ib}_{2}$ & $\mathrm{a}_{9} \mathrm{Sb}_{2}$ \\
\hline $\mathrm{a}_{3} \mathrm{Sb}_{1}$ & $\mathrm{a}_{10} \mathrm{Sb}_{1}$ & $\mathrm{a}_{3} \mathrm{Sb}_{2}$ & $\mathrm{a}_{10} \mathrm{Sb}_{2}$ \\
\hline $\mathrm{a}_{4} \mathrm{Sb}_{1}$ & $\mathrm{a}_{11} \mathrm{Sb}_{1}$ & $\mathrm{a}_{4} \mathrm{Ib}_{2}$ & $\mathrm{a}_{11} \mathrm{Sb}_{2}$ \\
\hline $\mathrm{a}_{5} \mathrm{Sb}_{1}$ & $\mathrm{a}_{12} \mathrm{Sb}_{1}$ & $\mathrm{a}_{5} \mathrm{Ib}_{2}$ & $\mathrm{a}_{12} \mathrm{Ib}_{2}$ \\
\hline $\mathrm{a}_{6} \mathrm{Sb}_{1}$ & $\mathrm{a}_{13} \mathrm{Sb}_{1}$ & $\mathrm{a}_{6} \mathrm{Sb}_{2}$ & $\mathrm{a}_{13} \mathrm{Sb}_{2}$ \\
\hline $\mathrm{a}_{7} \mathrm{Sb}_{1}$ & & $a_{7} S_{2}$ & \\
\hline \multicolumn{2}{|c|}{$\mathbf{a}_{\mathbf{k}} \boldsymbol{S b}_{3}$} & & $\mathbf{a}_{\mathbf{k}} \mathbf{S b}_{4}$ \\
\hline $\mathrm{b}_{3} \mathrm{Sa}_{1}$ & $\mathrm{a}_{8} \mathrm{Rb}_{3}$ & $\mathrm{~b}_{4} \mathrm{Sa}_{1}$ & $\mathrm{~b}_{4} \mathrm{Sa}_{8}$ \\
\hline $\mathrm{b}_{3} \mathrm{Sa}_{2}$ & $\mathrm{a}_{9} \mathrm{Sb}_{3}$ & $\mathrm{~b}_{4} \mathrm{Sa}_{2}$ & $\mathrm{~b}_{4} \mathrm{Sa}_{9}$ \\
\hline $\mathrm{a}_{3} \mathrm{Sb}_{3}$ & $\mathrm{a}_{10} \mathrm{Ib}_{3}$ & $\mathrm{~b}_{4} \mathrm{Sa}_{3}$ & $\mathrm{~b}_{4} \mathrm{Sa}_{10}$ \\
\hline $\mathrm{b}_{3} \mathrm{Sa}_{4}$ & $\mathrm{a}_{11} \mathrm{Sb}_{3}$ & $\mathrm{~b}_{4} \mathrm{Sa}_{4}$ & $\mathrm{~b}_{4} \mathrm{Sa}_{11}$ \\
\hline $\mathrm{b}_{3} \mathrm{Sa}_{5}$ & $\mathrm{a}_{12} \mathrm{Ib}_{3}$ & $\mathrm{~b}_{4} \mathrm{Sa}_{5}$ & $\mathrm{~b}_{4} \mathrm{Sa}_{12}$ \\
\hline $\mathrm{a}_{6} \mathrm{Sb}_{3}$ & $\mathrm{a}_{13} \mathrm{Rb}_{3}$ & $\mathrm{a}_{6} \mathrm{Ib}_{4}$ & $\mathrm{~b}_{4} \mathrm{Sa}_{13}$ \\
\hline $\mathrm{a}_{7} \mathrm{Sb}_{3}$ & & $\mathrm{a}_{7} \mathrm{Ib}_{4}$ & \\
\hline
\end{tabular}
(S) ou de indiferença (I).

Tabela 9: Relações Binárias a H b 
que consiste em alocar as ações do problema às categorias previamente definidas. Este processo, conhecido como procedimento de alocação, visa essencialmente analisar a maneira com que cada ação se compara com os perfis de referência que delimitam as categorias $C_{j}$. Esta comparação permite determinar a categoria em que cada ação deve ser alocada.

O procedimento de alocação foi iniciado com o ELECTRE TRI Pessimista ${ }^{5}$ (procedimento conjuntivo). Neste procedimento, a comparação se inicia com o a melhor ação de referência e prossegue para a ação imediatamente inferior, até a identificação da primeira ação de referência $b_{h}$, que é superada por $a$. Aloca-se, então, a ação $a$ à categoria que é limitada na parte inferior por essa ação de referência $b_{h}$. Logo após, passouse ao ELECTRE TRI Otimista (procedimento disjuntivo). Neste procedimento, a comparação de $a$ é iniciada com a pior ação de referência, passando-se à ação imediatamente superior, até se identificar a primeira ação de referência $\mathrm{b}_{\mathrm{h}}$ que supera $a$. A ação $a$ é então alocada à categoria que é limitada na parte superior pela ação de referência $b_{h}$.

Uma primeira conclusão sobre os dois procedimentos de alocação adotados é que eles atenderam à exigência de unicidade. Tal exigência determina que cada ação deve ser alocada a uma, e apenas uma, categoria $C_{k}$ construída no modelo. Com isso, não se verificou a ocorrência da dupla alocação de ações.

Para se determinar a recomendação final do trabalho, os dois procedimentos adotados foram extensivamente discutidos. Contudo, a alocação pessimista pôde finalmente prevalecer em função dos motivos descritos a seguir:

(1) Os

profissionais

envolvidos no trabalho julgaram que o ELECTRE TRI

Pessimista é mais consistente para o

\footnotetext{
5 Os procedimentos de alocação otimista e pessimista foram propostos por Yu (1992). ENGEVISTA, v. 6, n. 1, p. 4-18, abr. 2004
}

cenário

preponderantemente restritivo de recursos destinados aos custos com pessoal. Com isso, prevaleceu a noção de que na dúvida entre as categorias, as ações analisadas deveriam ser alocadas nas categorias mais baixas possíveis.

(2) Obteve-se consenso sobre a filosofia de alocação a ser adotada. Neste sentido, prevaleceu a noção de que, para estar alocada numa categoria, uma determinada unidade deveria, impreterivelmente, possuir características suficientemente fortes para superar a ação que delimita a parte inferior tal categoria. Desta forma, prevaleceu o procedimento de alocação mais rigoroso, onde a alocação em uma categoria só se justifica se uma unidade possuir condições suficientes para tal.

\subsection{Análises de Sensibilidade e de Robustez}

Após a alocação das unidades em suas respectivas categorias, as atenções voltaram-se para a execução das análises de sensibilidade e de robustez. Objetivouse nesta fase verificar de que forma as variações introduzidas nos parâmetros determinados no modelo influenciam os resultados obtidos. Com isso, os resultados das alocações otimista e pessimista foram considerados provisórios.

Tendo em vista todas as hesitações que surgiram durante a construção dos pseudo-critérios e dos valores que definiram a relação $S \mathrm{k}_{\mathrm{j}}, \mathrm{v}_{\mathrm{j}}$ e $\lambda$, as 
análises de sensibilidade e de robustez se mostraram imprescindíveis para se chegar a uma recomendação que pudesse ser considerada robusta. A principal questão que se impôs nesta etapa do trabalho era determinar se a conclusão sobre a alocação das unidades poderia ser considerada definitiva, a partir da verificação da estabilidade proporcionada por estas análises, ou, então, se deveria haver uma revisão de tal conclusão a partir dos novos valores introduzidos nos parâmetros.

As análises de sensibilidade e robustez foram estruturadas em duas etapas distintas: (1) introdução de duas famílias diferentes de limiares $\mathrm{q}_{\mathrm{j}}$ e $\mathrm{p}_{\mathrm{j}}$, assim como $\mathrm{k}_{\mathrm{j}}$ e $\mathrm{v}_{\mathrm{j}}$ nos 7 critérios adotados no estudo; (2) para os resultados provisórios e para os dois conjuntos de limiares diferentes, adotou-se valores de $\lambda$ iguais a 0,9 e 0,7 . É importante sinalizar que se adotou valores que pudessem ser considerados extremos, ou seja, os intervalos entre tais valores foram substancialmente aumentados, de maneira a se colocar em evidência a estabilidade dos resultados.

Os exames críticos empreendidos após a introdução das novas famílias, demonstraram os seguintes pontos:

- os resultados advindos dos parâmetros iniciais praticamente não sofreram alterações a partir das mudanças introduzidas nos limiares $\mathrm{q}_{\mathrm{j}}$ e $\mathrm{p}_{\mathrm{j}}$, assim como de $\mathrm{k}_{\mathrm{j}}$ e $\mathrm{v}_{\mathrm{j}}$;

- as mudanças geradas pela introdução de $\lambda$ $(0,9$ e 0,7$)$, proporcionaram sensíveis alterações na alocação das unidades (julgou-se

desnecessário examinar os resultados através da alocação otimista em função dos argumentos anteriormente colocados). Todavia, os efeitos de tais alterações não foram consideradas satisfatórios em virtude da excessiva concentração de unidades nas categorias intermediárias.

Portanto, a execução das análises de sensibilidade e de robustez puderam comprovar a estabilidade dos resultados inicialmente encontrados. Desta forma, pôde-se chegar a conclusão que os resultados parciais definidos a partir da família inicial de parâmetros, justificam a conclusão inicial do estudo.

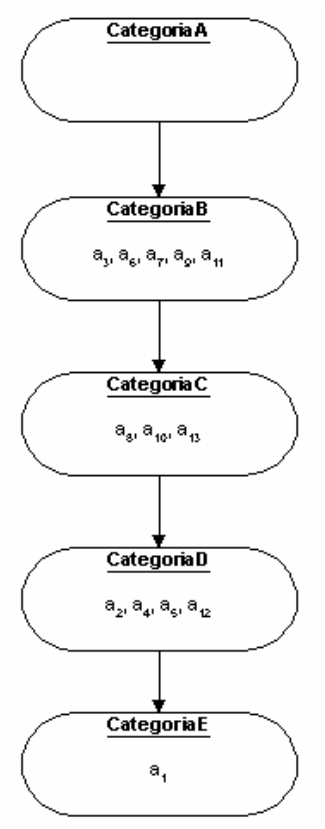




\section{Figura 1: Classificação Final das ações nas Categorias}

\section{CONCLUSÕES E RECOMENDAÇÕES}

Após o desenvolvimento do estudo e de sua efetiva aplicação, diversos benefícios puderam ser identificados pelos profissionais que tiveram, de alguma forma, participação no trabalho. A seguir, abordaremos as principais conclusões que evidenciaram os ganhos proporcionados pelo estudo.

Inicialmente, o trabalho veio satisfazer a necessidade de estruturar as informações da área de $\mathrm{RH}$ de forma lógica e organizada. Paradoxalmente, a despeito da ampla gama de informações de pessoal existente na empresa, apenas um limitado número era efetivamente utilizado nos processos decisórios de gestão de pessoas. Suas decisões eram demasiadamente simples e pouco abrangentes. $\mathrm{O}$ estudo veio oferecer um meio de estruturar estas informações no sentido de proporcionar conhecimento a respeito do perfil, características e necessidades de cada órgão da companhia. Com isso, apesar do trabalho ter se concentrado no planejamento de custos de pessoal, tais conhecimentos passaram a ser utilizados em outros processos da Diretoria de RH.

Outra importante contribuição identificada foi a facilidade de criação e análise de alternativas. O trabalho contribuiu para que os profissionais envolvidos no estudo fossem mais criativos na busca de alternativas ou soluções para o problema. Com efeito, a medida que o projeto foi transcorrendo, novas dimensões e critérios foram surgindo, refletindo o caráter construtivista da modelagem da decisão. Adicionalmente, a análise de robustez propiciou que se afinasse os perfis das categorias consideradas criando, assim, a sensação de que se tinha esgotado todas as possibilidades de entendimento do problema.

Finalmente, o processo de comunicação foi substancialmente aprimorado. A modelagem dos objetivos e das preferências promoveu e facilitou a comunicação e a integração entre aqueles envolvidos ou afetados pelo planejamento. Este estudo desenvolveu um "quadro de pensamento" muito bem estruturado, garantindo que a lógica adotada fosse compreendida por todos. Ou seja, podemos dizer que foi criada uma "linguagem" comum para o processo decisório. Através de uma metodologia que formalizou e estruturou múltiplos objetivos, o estudo serviu também para facilitar não apenas o entendimento, mas também a defesa de pontos de vista adotados, buscando legitimar a decisão que foi tomada. Tudo isso contribuiu para a geração de um clima de comprometimento e aceitação nos órgãos. As decisões foram muito bem fundamentadas, o que reforçou a credibilidade do processo

A fim de compartilhar as lições apreendidas com o desenvolvimento deste estudo, é importante tecer algumas recomendações para fututos trabalhos. Inicialmente, é fundamental que se tenha uma abordagem fundamentada no envolvimento e participação de todos os atores importantes no desenvolvimento de uma decisão. Tal estratégia de atuação visa, antes de mais nada, promover um sentimento de "paternidade" entre aqueles que são, em última instância, responsáveis pela execução e conseqüências da decisão. Pôde-se constatar que os profissionais de $\mathrm{RH}$ e de outras áreas sentiram-se muito mais confiantes em aplicar a metodologia desenvolvida a partir da participação ativa na construção do modelo. Ou seja, é fundamental que eles se sintam donos da decisão, de modo que o trabalho seja realmente fruto de suas convicções, experiências, sentimentos e valores.

Por fim, é da mais alta importância que se faça uma cuidadosa investigação do contexto da decisão antes de se iniciar a 
construção da modelagem de decisão baseado em técnicas de AMD. Conforme colocado anteriormente, os estudos comparativos entre as diversas modalidades de AMD, desmontram que não existe nenhuma metodologia que se destaca das outras em todos os contextos de decisão envolvendo múltiplos

\section{BIBLIOGRAFIA}

GOMES, L.F.A.M., ARAYA, M.C.G. e CARIGNANO, C. (2003) Tomada de Decisões em Cenários Complexos. São Paulo: Thomson.

LARICHEV, Oleg I. e OLSON, David L. (2001) Multiple Criteria Analysis in Strategic Siting Problems. Dordrecht: Kluwer Academic Publishers.

ROY, Bernard (1968) Méthodologie Multicritère d'Aide à la Décision. Paris: Economica.

ROY, Bernard e BOUYSSOU, Denis (1993) Aide Multicritère à la Décision - Méthodes et Cas. Paris: Economica

YU, W. (1992) Aide Multicritère à la Décision dans le Cadre de la Problématique du Tri: Concepts, Méthodes et Applications. Tese (Doutorado). - Universidade de Paris IX-Dauphine. critérios. Portanto, é fundamental empreender uma minuciosa investigação das principais características da decisão de modo a se certificar que o modelo escolhido seja efetivamente adequado para o problema. 